\title{
Model Energy Landscapes of Low-Temperature Fluids: Dipolar Hard Spheres
}

\author{
Dmitry V. Matyushov \\ Department of Physics and Astronomy and Center for Biophysics, \\ Arizona State University, PO Box 871604, Tempe, AZ 85287-1604
}

(Dated: November 3, 2018)

\begin{abstract}
An analytical model of non-Gaussian energy landscape of low-temperature fluids is developed based on the thermodynamics of the fluid of dipolar hard spheres. The entire excitation profile of the liquid, from the high temperatures to the point of ideal-glass transition, has been obtained from the Monte Carlo simulations. The fluid of dipolar hard spheres loses stability when reaching the point of ideal-glass transition transforming via a first-order transition into a columnar liquid phase of dipolar chains locally arranged in a body-centered tetragonal order.

PACS numbers: 64.70.Pf,65.40.Gr,66.20.+d
\end{abstract}

Thermodynamic and dynamic properties of supercooled liquids are often related to the excess of configurations they possess relative to the crystalline phase [1]. The number of states of the liquid state is reflected by the configurational entropy $S_{c}(T)$ conventionally defined [2, 3, 4] as the logarithm of the density of states $\Omega(E)$ taken at the energy equal to the internal energy $E(T)$ at temperature $T$ :

$$
S_{c}(T)=\ln [\Omega(E(T))] .
$$

The energy integral of the density of states and the Boltzmann factor provide the canonical configuration integral

$$
Z(\beta)=\int \Omega(E) e^{-\beta E} d E
$$

where $\beta$ is the inverse temperature.

Stillinger [5, 6] gave an alternative definition of the configurational entropy in terms of the density of states $\Omega_{\phi}(E)$ of inherent structures, i.e. minima of the total energy of the system as a function of its all translational an rotational degrees of freedom (energy landscape). The canonical configuration integral is now given by integrating over the basin depths $\phi$ and the free energy of interbasin vibrations $F_{v}(\phi)$, which is a weak, approximately linear, function of $\phi[7,8]$

$$
Z(\beta)=\int d \phi \Omega_{\phi}(\phi) e^{-\beta \phi-\beta F_{v}(\phi)} .
$$

Because of the central role of the configurational entropy in early theories of viscous liquids by Adam, Gibbs, and DiMarzio [9, 10], and more recent random first-order transition models by Wolynes and co-workers [1], much effort has been invested in calculating the Stillinger configurational entropy, $S_{c}^{\phi}(T)=\ln \left[\Omega_{\phi}(\phi(T))\right]$, from computer simulations of model fluids [7, 8,12$]$. A general

*E-mail dmitrym@asu.edu. connection between $S_{c}(T)$ and $S_{c}^{\phi}(T)$ is, however, still unclear.

Analytical modeling of the landscape of supercooled liquids is to a large extent based on the ideas advanced for spin glasses with quenched disorder [13, 14]. The most prominent role in application to structural glasses is played by Derrida's random-energy, or Gaussian landscape, model (REM) 15]. Computer simulations, mostly limited to temperatures above the mode-coupling critical temperature, basically support the REM, in particular the prediction of the $1 / T$ falloff of the average basin energy $\phi(T)$ from its high-temperature plateau [7, 8]. However, general theoretical arguments [6, 16] and recent simulations [12] suggest that the low-energy portion of the Gaussian landscape might be inaccurate. In particular, combinatorial arguments suggest that the derivative of the enumeration function, $\sigma_{\phi}(\phi)=N^{-1} \ln \left[\Omega_{\phi}(\phi)\right]$, approaches infinity at the point of ideal glass transition when the system runs out of configurations and $\sigma_{\phi}\left(\phi_{I G}\right)=0$ ( $N$ is the number of liquid particles). This infinite derivative eliminates the ideal glass transition at a positive temperature [6]. A phenomenological description, patching together logarithmic and Gaussian enumeration functions, was suggested to provide a correct low-energy portion of $\sigma_{\phi}(\phi)$ [17].

Generally, unlike the case of spin glasses, there has been a lack of simple, solvable models of structural glasses. In this Letter we propose a new model of nonGaussian landscape of low-temperature fluids with no quenched disorder. Our derivation is based on the established thermodynamics of the model fluid of dipolar hard spheres (DHS) [18]. This monoatomic glass former (in contrast to binary mixtures used in many recent simulations $7,[8]$ ) is long known to resist phase transformations. This fluid lacks the liquid-vapor transition decomposing instead into low-density dipolar chains 19, 20]. Upon cooling, dipolar hard spheres transform into a ferroelectric fluid [21], but the ferroelectric phase is stabilized by tin-coil boundary conditions used in simulations and can be prevented by using a low dielectric constant for the reaction-field or Ewald corrections for the cutoff of dipo- 
lar interactions 22]. This strategy has been employed in this study. We have carried out the Monte Carlo (MC) simulations of the DHS fluid within the standard NVT Metropolis protocol and the reaction-field cutoff corrections. The reaction-field dielectric constant of $\epsilon_{\mathrm{RF}}=10$, below the lowest value $\simeq 18$ permitting the ferroelectric phase 22], has allowed us to eliminate the transition to liquid ferroelectric. The initial configuration was set up as a face-centered cubic lattice of 108 dipolar spheres. The cubic simulation box also helps to suppress crystallization of dipoles which do not favor highly symmetric lattice structures 23, 24]. The data were collected for $(2-10) \times 10^{7}$ MC cycles.

Apart from avoiding crystallization, a significant advantage of the DHS fluid is the existence of a simple analytical form for the free energy $\beta F(\beta)=-\ln [Z(\beta)]$. The Padé truncation of perturbation series suggested by Stell and co-workers [25] turned out to be very successful when tested against simulations [18]. The free energy of dipolar hard spheres depends on two parameters, the reduced density $\rho^{*}=\rho \sigma^{3}$ and the reduced temperature $T=k_{\mathrm{B}} T \sigma^{3} / m^{2}$. Here, $\rho$ is the number density, $\sigma$ is the hard-sphere diameter, and $m$ is the dipole moment. All calculations and simulations here have been done at constant volume with $\rho^{*}=0.8$ thus reducing the number of variables to one.

Stell's Padé solution for $F(\beta)$ is

$$
\beta F(\beta)=N f(\eta)-\frac{A \beta^{2}}{1+b \beta} .
$$

Here $f(\eta)$ is the reduced free energy of the fluid of hard spheres with zero dipole moment as a function of the packing density $\eta=(\pi / 6) \rho^{*}$. For the Carnahan-Starling equation of state, on has $f(\eta)=\left(4 \eta-3 \eta^{2}\right) /(1-\eta)^{2}$. $\beta=$ $1 / T$ in Eq. (4) is given in reduced units and thus the coefficients $A=N a$ and $b$ depend only on the liquid density through two-particle and three-particle perturbation integrals, $a=\left(\rho^{*} / 6\right) I^{(2)}\left(\rho^{*}\right), b=\left(\rho^{*} / 9\right) I^{(3)}\left(\rho^{*}\right) / I^{(2)}\left(\rho^{*}\right)$, tabulated by Larsen et al 25$]$.

Following Freed [4], the density of states can be obtained by inverse Laplace transformation of Eq. (2) in which we use $\beta F(\beta)$ in the Padé form given by Eq. (4). The inverse Laplace transform is calculated by expanding $\exp (-\beta F(\beta))$ in powers of the second summand in Eq. (4) and performing pole integration. The result can be converted to a closed-form equation:

$$
\begin{aligned}
\Omega(e)=(b \sqrt{1+e / c})^{-1} & \exp [N(f(\eta)-e / b-2 c / b)] \\
& I_{1}(2(c / b) N \sqrt{1+e / c}),
\end{aligned}
$$

where $c=a / b, I_{1}(x)$ is the first-order modified Bessel function, and $e=E \sigma^{3} / \mathrm{Nm}^{2}$ is the reduced internal energy per particle. Since the argument of the Bessel function in Eq. (5) is proportional to the number of particles $N$, its thermodynamic-limit expansion gives the following expression for the enumeration function

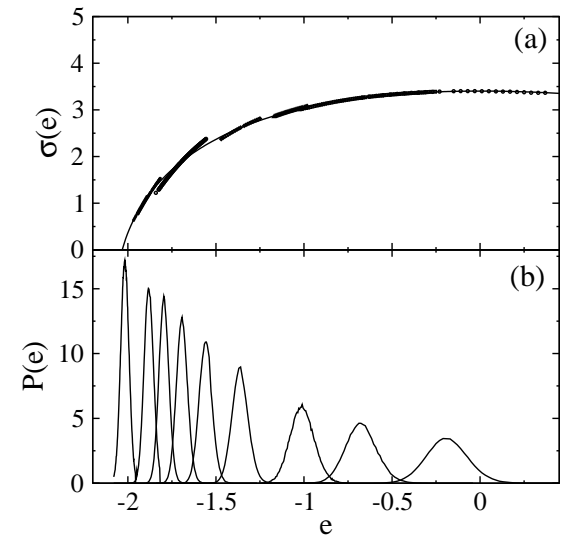

FIG. 1: Enumeration function $\sigma(e)$ (a) and distribution function $P(e)(\mathrm{b})$ of the fluid of dipolar hard spheres at $\rho^{*}=0.8$ and the temperatures $T$ (from left to right in (b)): 0.125 , $0.167,0.2,0.25,0.33,0.5,1.0,2.0,10.0$. The simulation points in (a) are obtained from $P(e)$ within $95 \%$ of the maximum probability at each temperature.

$$
\begin{aligned}
& \sigma(e)=N^{-1} \ln [\Omega(e)]: \\
& \sigma(e)=f(\eta)-b^{-1}(\sqrt{c+e}-\sqrt{c})^{2} .
\end{aligned}
$$

The enumeration function in Eq. (6) is Gaussian near its top, $e \simeq 0$, where the dipole-dipole interactions are insignificant and the limit of a hard-sphere fluid is reached. It deviates from the parabolic shape in its low-energy wing, in particular close to the low-energy cutoff at $e_{0}=-c$ (Fig. 17). Depending on the parameters, Eq. (6) gives two possible resolutions of the entropy crisis of low-temperature fluids [1]. When $f(\eta)-c / b \geq 0$, the enumeration function has an infinite derivative at $e_{0}$ and, according to Stllinger's arguments [6], there is no ideal glass transition. When $f(\eta)-c / b$ is strictly positive, the entropy is non-zero at the cutoff energy, a situation observed for network glass formers [26]. Finally, when $f(\eta)-c / b<0$, the ideal glass arrest $\sigma\left(e_{I G}\right)=0$ happens before the cutoff is reached, and the temperature of idealglass transition $T_{I G}, e_{I G}=e\left(T_{I G}\right)$ is positive. The use of Carnahan-Starling hard-sphere free energy, and perturbation integrals from Ref. 25 gives $f(\eta)-c / b=-1.19$ at $\rho^{*}=0.8$ and $T_{I G} \simeq 0.073$.

The enumeration function from Eq. (6) is compared to the results of MC simulations in Fig. 17. The simulation points were obtained by patching together central parts (within $95 \%$ of the maximum) of the distribution functions $P(e) \propto \exp (N(\sigma(e)-\beta e))$ at different temperatures. Since $P(e)$ defines $\sigma(e)$ up to a constant, this comparison only serves to show the non-Gaussian shape of the curve. This non-Gaussian form of $\sigma(e)$ forces the width of $P(e)$ to decrease with cooling (Fig. 10). This behavior is quite distinct from the prediction of the Gaussian landscape model in which the width is temperatureindependent [15].

The temperature dependence of the width is directly 


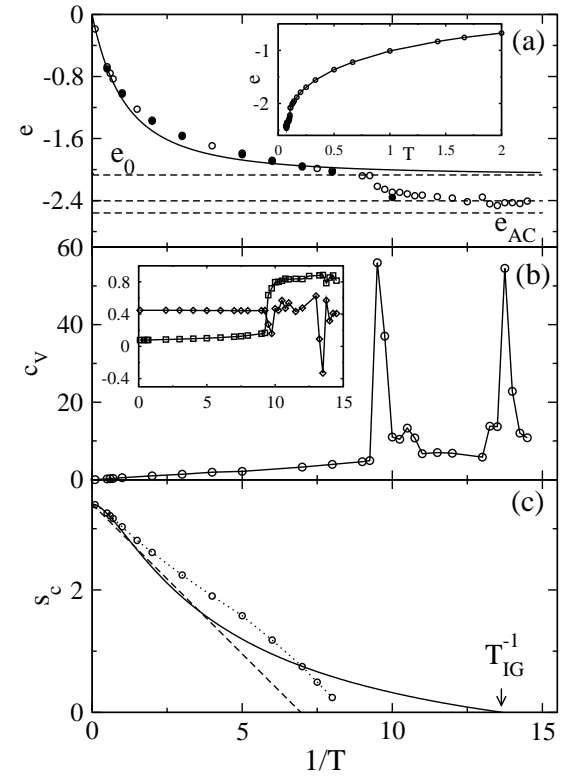

FIG. 2: Average energy $e(T)(\mathrm{a})$, heat capacity $c_{V}$ (b), and the configurational entropy $s_{c}$ (c) vs $1 / T$. The points in (a) are the results of MC simulations: open points for the internal energies and close points for the energies of inherent structures. The solid line in (a) shows the Padé approximation of Eq. (8). The dashed horizontal lines show the cutoff energy $e_{0}=-2.07$, the energy of non-interacting dipolar chains $e=-2.4$ (middle line), and the energy of the closed-packed fcc antiferroelectric crystal $e_{A C}=-2.56$. The inset in (a) shows $e(T)$ from simulations, the inset in (b) shows the nematic order parameter $P_{2}$ (squares) and the Binder parameter $M_{B}$ (diamonds). The solid line in (c) is the configurational entropy $s_{c}(T)=\sigma(e(T))$ in the Padé approximation [Eqs. (6) and (8)]. The dots show the configurational entropy [Eq. (6)] with $e(T)$ from simulations. The dashed line in (c) is the thermodynamic excess entropy over that of the ideal gas calculated by temperature integration of simulated $c_{V}(T)$.

related to the heat capacity since

$$
P(e) \propto \exp \left[-\frac{(\delta e)^{2}}{2 c_{V} T^{2}}\right]
$$

where $\delta e$ is the energy fluctuation and $c_{V}$ is the excess constant-volume heat capacity over that of the ideal gas. The high-temperature portion of the heat capacity, corresponding to the distributions shown in Fig. 1b, scales linearly with the inverse temperature, $c_{V} \propto 1 / T$ (Fig. $2 \mathrm{~b}$ ). This hyperbolic temperature scaling, often documented for structural glass formers at constant pressure [27], results in a linear temperature scaling of the squared width when the distribution $P(e)$ is fitted to a Gaussian function. This behavior was predicted by models of supercooled liquids in terms of configurational excitations [16].

The calculation of the energies of inherent structures by conjugate gradient minimization of configurations along simulated trajectories results in energies $\phi(T)$ just slightly below $e(T)$ (closed points in Fig. 2a). This means that the long-range dipolar forces produce essentially a mean-field potential for the local translations and rotations and Eq. (6) for the density of internal energies can be used for the density of inherent structures as well, $\Omega(e) \simeq \Omega_{\phi}(e)$.

The density of states from simulations approaches the ideal-glass state even steeper than Eq. (6) (Fig. 17a). This trend results in a slightly steeper temperature drop of $e(T)$ from simulations compared to the result of the Padé approximation (Fig. 2a):

$$
e(T)=-c+c(1+b / T)^{-2} .
$$

In particular, instead of leveling off while approaching the cutoff energy $e_{0}=-2.07$, the system undergoes a first-order liquid-liquid transition (see below) accompanied by a discontinuous drop of the internal energy and a sharp peak in the heat capacity (Fig. 2a,b). The orientational structure of the fluid also changes as indicated by a step-wise increase in the nematic order parameter $P_{2}$ calculated as the largest eigenvalue of the $\mathbf{Q}$-tensor

$$
\mathbf{Q}=(2 N)^{-1} \sum_{j}\left(3 \hat{\mathbf{e}}_{j} \hat{\mathbf{e}}_{j}-\mathbf{I}\right),
$$

where $\hat{\mathbf{e}}_{j}$ is a unit vector along the dipole of particle $j$. The ferroelectric order parameter $P_{1}$ (normalized total dipole moment of the liquid $\mathbf{M}$ ) remains close to zero indicating that the liquid remains unpolarized. The Binder parameter [28], $M_{B}=1-\left\langle\mathbf{M}^{4}\right\rangle / 3\left\langle\mathbf{M}^{2}\right\rangle^{2}$, also shows downward spikes pointing to first-order phase transitions (inset, Fig. 2b). The internal energy at the first spike of $c_{V}$ drops from the level $e_{0}$ to the energy approximately equal to the energy of non-interacting dipolar chains 29] $e=-2.40$ (middle dashed line in Fig. 2a). This energy is slightly above the energy of close-packed antiferroelectric fcc crystal $e_{A C}=-2.56[30]$.

The configurational entropy per particle $s_{c}(T)=$ $\sigma(e(T))$ from Padé thermodynamics [Eqs. (6) and (8)] deviates from the hyperbolic functions at low temperatures approaching the ideal glass transition at a positive temperature (Fig. 28). In contrast, when $e(T)$ from simulations is substituted into Eq. (6), the decay of $s_{c}(T)$ is faster, reaching the ideal-glass state near the point of the first-order transition. The DHS fluids thus loses stability when it runs out of configurations when approaching the ideal-glass transition and transforms into a different thermodynamic state via a first-order transition.

The properties of the low-temperature fluid phase are peculiar. Both the density $\left(\propto\left\langle\left|\rho_{k}\right|^{2}\right\rangle\right)$ and polarization $\left(\propto\left\langle\left|M_{k}\right|^{2}\right\rangle\right)$ structure factors do not show crystalline spatial or orientational order. However, snapshots of simulated configurations (not shown here) indicate the existence of chains of dipoles aligned head-to-tail. These chains are persistent for $(5-20) \times 10^{3} \mathrm{MC}$ cycles resulting in the overall slow convergence of simulations. The chains of parallel dipoles are arranged in bundles with the locally body-centered tetragonal (bct) structure in which the two chains are displaced relative to each other by the 


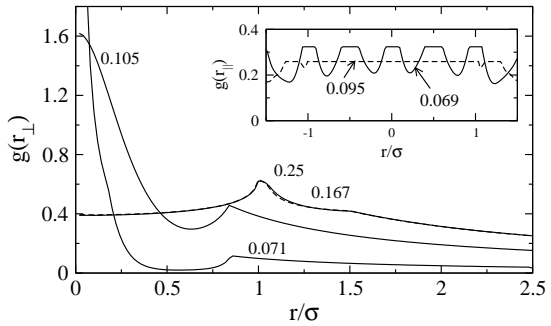

FIG. 3: Pair distribution function of transverse interparticle separations (relative to the nematic director) at temperatures indicated in the plot. The high-temperature results for $T=0.167$ (dashed line) and $T=0.25$ almost coincide on the scale of the plot. The distribution of distances parallel to the director in the inset shows the transition to the smectic phase of parallel chains with the local bct order.

hard-sphere radius. This arrangement has the lowest energy for fluids forming columnar phases of parallel dipolar chains [29, 31]. A body-centered orthorombic lattice was suggested as the ground state for Stockmayer ferroelectrics [23]. In the present case, all the chain bundles are oriented along the same director, but the net moment is compensated among the oppositely oriented bundles.

The columnar structure is well seen in the pair correlation function of transverse separations, which is very sensitive to columnar order [32] (Fig. 3). At the phase transition, a peak at zero transverse projection indicates the emergence of the columnar phase. At the same time, a smaller peak, present at $r / \sigma=1$ in the isotropic dipolar fluid, shifts to $r / \sigma=\sqrt{3} / 2$ characteristic of the closest bct distance [29]. The one-dimensional dipolar chains experience strong Landau-Peierls fluctuations with the orthogonal mean-square displacement scaling as $\left\langle r_{\perp}^{2}\right\rangle \propto l T$ 33], where $l$ is the average length of the chain. The fits of the initial portion of $g\left(r_{\perp}\right)$ result is almost invariant $\left\langle r_{\perp}^{2}\right\rangle \simeq 0.07 \sigma^{2}$ suggesting that the length of the chains increases as $1 / T$.

The nematic columnar phase transforms into a smectic phase with further cooling as indicated by the second peak of the heat capacity, a downward spike of the Binder parameter (Fig. 2b), and the appearance of clear density modulation in the distribution function of molecular separations parallel to the director (inset in Fig. 3).

In conclusion, a new model of thermodynamics of lowtemperature fluids offers a description of non-Gaussian landscape in a good agreement with simulations. For the parameters of the DHS fluids studied here the model predicts the excitation profile $e(T)$ to terminate at the ideal-glass transition. The DHS fluid nearly avoids this point through a first-order transition to a fluid phase with columnar order.

This research was supported by the the Air Force (FA9550-06-C-0084) and NSF (CHE-0616646).
[1] C. A. Angell, K. L. Ngai, G. B. McKenna, and S. W. Martin, J. Appl. Phys. 88, 3113 (2000).

[2] I. M. Lifshitz, A. Y. Grosberg, and A. R. Khokhlov, Rev. Mod. Phys. 50, 683 (1978).

[3] J. D. Bryngelson and P. G. Wolynes, Proc. Natl. Acad. Sci. 84, 7524 (1987).

[4] K. F. Freed, J. Chem. Phys. 119, 5730 (2003).

[5] F. H. Stillinger and T. A. Weber, Phys. Rev. A 25, 978 (1982).

[6] F. H. Stillinger, J. Chem. Phys. 88, 7818 (1988).

[7] S. Büchner and A. Heuer, Phys. Rev. E 60, 6507 (1999).

[8] S. Sastry, Nature 409, 164 (2001).

[9] J. H. Gibbs and E. A. D. Marzio, J. Chem. Phys. 28, 373 (1958).

[10] G. Adam and J. H. Gibbs, J. Chem. Phys. 43, 139 (1965).

[11] X. Xia and P. G. Wolynes, Proc. Nat. Acad. Sci. 97, 2990 (2000).

[12] A. J. Moreno, I. Saika-Voivod, E. Zaccarelli, E. L. Nave, S. V. Buldyrev, P. Tartaglia, and F. Sciortino, J. Chem. Phys. 124, 204509 (2006).

[13] M. Mezard, G. Parisi, and M. Virasoro, Spin Glass Theory and Beyond (World Scientific, Singapore, 1987).

[14] T. R. Kirkpatrick, D. Thirumalai, and P. G. Wolynes, Phys. Rev. A 40, 1045 (1989).

[15] B. Derrida, Phys. Rev. B 24, 2613 (1981).

[16] D. V. Matyushov and C. A. Angell, J. Chem. Phys. 123, 034506 (2005); cond-mat/0612627.

[17] P. G. Debenedetti, F. H. Stillinger, and M. S. Shell, J. Phys. Chem. B 107, 14434 (2003).
[18] C. G. Gray and K. E. Gubbins, Theory of Molecular Liquids (Clarendon Press, Oxford, 1984).

[19] J. J. Weis and D. Levesque, Phys. Rev. Lett. 71, 2729 (1993).

[20] M. E. van Leeuwen and B. Smit, Phys. Rev. Lett. 71, 3991 (1993).

[21] D. Wei and G. N. Patey, Phys. Rev. Lett. 68, 2043 (1992).

[22] D. Wei, G. N. Patey, and A. Perera, Phys. Rev. E 47, 506 (1993).

[23] G. T. Gao and X. C. Zeng, Phys. Rev. E 61, R2188 (2000).

[24] B. Groh and S. Dietrich, Phys. Rev. E 63, 021203 (2001).

[25] B. Larsen, J. C. Rasaiah, and G. Stell, Mol. Phys. 33, 987 (1977).

[26] A. Saksaengwijit, J. Reinisch, and A. Heuer, Phys. Rev. Lett. 93, 235701 (2004).

[27] R. Richert and A. C. Angell, J. Chem. Phys. 108, 9016 (1998).

[28] M. S. S. Challa, D. P. Landau, and K. Binder, Phys. Rev. B 34, 1841 (1986).

[29] R. Tao and J. M. Sun, Phys. Rev. Lett. 67, 398 (1991).

[30] J. M. Luttinger and L. Tisza, Phys. Rev. 70, 954 (1946).

[31] A.-P. Hynninen and M. Dijkstra, Phys. Rev. Lett. 94, 138303 (2005).

[32] D. Wei, Phys. Rev. E 49, 2454 (1994).

[33] P. G. de Gennes and P. A. Pinkus, Phys. Kondens. Materie 11, 189 (1970). 